\title{
INTERPOLATION INEQUALITIES IN WEIGHTED SOBOLEV SPACES
}

\author{
SERENA BOCCIA AND LOREDANA CASO
}

Abstract. In this paper we prove some interpolation inequalities between functions and their derivatives in the class of weighted Sobolev spaces defined on unbounded open subset $\Omega \subset \mathbb{R}^{n}$.

Mathematics subject classification (2000): 26D10, 47A30, 46E30.

Key words and phrases: Interpolation inequalities, weighted spaces.

\section{REFERENCES}

[1] R. A. AdAms, Sobolev Spaces, Academic Press, New York (1975).

[2] R. C. BRown AND D. B. Hinton, Weighted interpolation inequalities and embenddings in $\mathbb{R}^{n}$, Canad. J. Math. 42 (1990), 959-980.

[3] L. Caffarelli, R. Kohn And L. Nirenberg, First order interpolation inequalities with weights, Compos. Math. 3 (1984), 259-275.

[4] L. CAso And M. TransiRico, Some remarks on a class of weight functions, Comment. Math. Univ. Carolinae 37 (1996), 469-477.

[5] E. Gagliardo, Proprietà di alcune classi di funzioni in più variabili, Ricerche Mat. 7 (1958), $102-137$.

[6] A. Kovalevsky AND F. Nicolosi, A weighted interpolation inequality of the Nirenberg - Gagliardo kind, Nonlinear Anal. 36 (1999), 269-273.

[7] C. MirandA, Su alcune disuguaglianze integrali, Atti Accad. Naz. Lincei Mem. Cl. Sci. Fis. Mat. Natur. 7 (1963), 1-14.

[8] L. NiRenberG, On elliptic partial differential equations, Ann. Scuola Norm. Sup. Pisa, 13 (1959), $116-162$.

[9] M. TROISI, Teoremi di inclusione negli spazi di Sobolev con peso, Ricerche Mat. 18 (1969), 177-189.

[10] M. Troisi, Su una classe di funzioni peso, Rend. Accad. Naz. Sci. XL Mem. Mat. 10 (1986), 141-152.

[11] M. Troisi, Su una classe di spazi di Sobolev con peso, Rend. Accad. Naz. Sci. XL Mem. Mat. 10 (1986), 177-189. 